\title{
TENSOR PRODUCTS OF DIVISION RINGS AND FINITE GENERATION OF SUBFIELDS
}

\author{
RICHARD RESCO, LANCE W. SMALL ${ }^{1}$ AND ADRIAN R. WADSWORTH ${ }^{1}$
}

\begin{abstract}
Let $D$ be a division algebra over a field $k$. It is shown that if $D \otimes_{k} D^{0}$ is Noetherian, then every commutative subfield of $D$ containing $k$ is finitely generated over $k$. This theorem applies to $D_{n}$, the quotient division algebra of the $n$th Weyl algebra, and also to a number of other standard examples of nonalgebraic division algebras.
\end{abstract}

If the division ring $D$ is finite dimensional over its center $k$, the (commutative) subfields of $D$ play a fundamental role in the structure of $D$. By contrast, if $D$ is not finite dimensional over $k$, very little is known about the subfields of $D$. The type of pathology that can arise is illustrated by examples of $\mathbf{M}$. Smith [7, pp. 162-163]: She showed that there is a division algebra $D$ with center $k$, containing two maximal subfields whose respective transcendence degrees are any two prescribed cardinal numbers.

For many of the familiar nonalgebraic division algebras $D$, insight into the subfields $L$ of $D$ can be obtained by use of the tensor product. In [4] it was shown that dimension properties of the rings $D \otimes_{k} k\left(x_{1}, \ldots, x_{n}\right)$ can give an upper bound on the transcendence degree of $L$ over $k$. Here we consider $D \otimes_{k} D^{0}$, where $D^{0}$ denotes the opposite algebra of $D$. Our basic result, Theorem 3, has a deceptively easy proof. It is worth noting that the consequences on finite generation of subfields given in Theorems 4 and 5 are new, and are results which seem completely inaccessible by direct computation.

Throughout this paper, $k$ denotes a field and tensor products, when not specifically marked, are taken relative to $k$. The term "Noetherian" means left Noetherian and right Noetherian.

The approach to proving Theorem 3 is faithfully flat descent from a tensor product of division rings to a tensor product of fields. The applicability of this technique to problems in division algebras was first noted in [5]. We need a result on fields which is isolated as Proposition 1. A proof of this was recently given by Vámos.

Proposition 1. Let $L$ be an extension field of $k$. Then $L \otimes L$ is Noetherian if and only if $L$ is finitely generated over $k$.

Received by the editors July 25, 1978 and, in revised form, October 5, 1978.

AMS (MOS) subject classifications (1970). Primary 16A40; Secondary 16A46.

Key words and phrases. Division rings, finitely generated subfields, Weyl algebras.

${ }^{1}$ Research supported in part by the National Science Foundation. 
Proof. See [8].

Before proceeding to the proof of Theorem 3, we make a direct application of Proposition 1 to a problem in rings with polynomial identity. It was proven in [6] that if the prime Noetherian PI-ring $R$ is finitely generated over a central subfield $k$, then $R \otimes R^{0}$ is Noetherian. We give an example here which shows that this fails without the assumption of finite generation.

EXAMPLE 2. Let $k$ be an arbitrary field, $A=k\left[y_{1}, y_{2}, \ldots\right]$ a polynomial ring over $k$ in countably many indeterminates, and let $K$ be the field of fractions of $A$. Let $\Sigma$ denote the permutation group of the set of positive integers and let $\Sigma$ act on $A$ and on $K$ by permuting the indeterminates. Let $\alpha, \beta \in \Sigma$ be the transpositions

$$
\alpha=(1,2)(3,4)(5,6) \ldots, \quad \beta=(2,3)(4,5)(6,7) \ldots,
$$

and put $\gamma=\beta \alpha$. While $\beta$ and $\alpha$ are each of order two, $\gamma$ is the infinite cycle

$$
\gamma=(\ldots, 8,6,4,2,1,3,5,7, \ldots) \text {. }
$$

If $K_{1}=K^{\alpha}$ is the fixed field of $\alpha$ and $K_{2}=K^{\beta}$ is that of $\beta$, then $K_{1} \cap K_{2} \subset$ $K^{\gamma}=k$. The last equality follows easily from the "lowest terms" representation, unique up to units, of an element of the quotient field of a unique factorization domain. We thus obtain a tower of fields

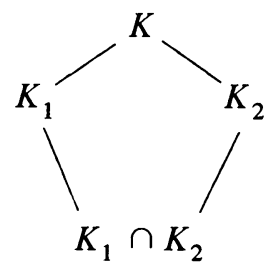

where $K$ has dimension two over $K_{1}$ and over $K_{2}$, but is not even finitely generated over the intersection.

Now let $K[x]$ be the ring of polynomials over $K$ and let $K_{i}+x K[x]$, $i=1,2$, denote the subring of $K[x]$ consisting of those polynomials whose constant term lies in $K_{i}$. In the ring of $2 \times 2$ matrices over $K[x]$ let $R$ be defined by

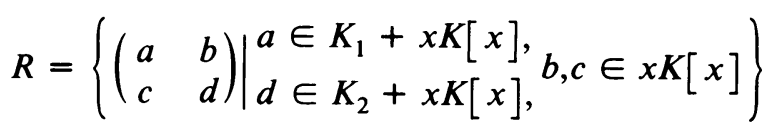

As is easily verified $R$ is a prime Noetherian PI-ring with center $C \simeq k+$ $x K[x]$. There is a surjective $C$-algebra homomorphism $R \rightarrow K_{1}$, obtained by mapping each matrix to its upper left entry, then reducing $\bmod x K[x]$. This map induces a ring epimorphism $R \otimes_{C} R^{0} \rightarrow K_{1} \otimes_{C} K_{1} \simeq K_{1} \otimes_{k} K_{1}$. Proposition 1 says that the image is not Noetherian; hence $R \otimes_{C} R^{0}$ cannot be Noetherian.

We note in passing that $R \otimes_{C} R^{0}$ is a semiprime ring, but is not prime. 
THEOREM 3. Let $D$ be a division algebra over $k, L \supset k$ a commutative subfield of $D$ :

(i) $D \otimes L$ is Noetherian if and only if $L$ is a finitely generated extension of $k$;

(ii) If $D \otimes D^{0}$ is Noetherian then $L$ is finitely generated over $k$.

Proof. (i) Suppose $D \otimes L$ is Noetherian. Since $D$ is a (left, say) vector space over $L, D \otimes L$ is a free $(L \otimes L)$-module. This implies that $D \otimes L$ is a left faithfully flat extension of $L \otimes L$ [2, pp. 50-52], and that $I(D \otimes L) \cap L$ $\otimes L=I$ for any ideal $I$ of $L \otimes L$. Thus $L \otimes L$ is Noetherian and, by Proposition $1, L$ is finitely generated. Conversely, if $L$ is finitely generated and $\left\{t_{1}, \ldots, t_{n}\right\}$ is a transcendence base for $L$ over $k$, then $D \otimes k\left(t_{1}, \ldots, t_{n}\right)$ is isomorphic to a localization of a polynomial ring over $D$ and is Noetherian. As $D \otimes L$ is a finite module over this ring, it too is Noetherian.

(ii) If $D \otimes D^{0}$ is Noetherian then by faithful flatness $D \otimes L$ is Noetherian. Now apply (i).

We first apply Theorem 3 to the quotient division ring of the Weyl algebra.

THEOREM 4. Let $k$ be a field of characteristic zero, $A_{n}=A_{n}(k)$ the Weyl algebra over $k$ and $D_{n}$ the quotient division ring of $A_{n}$. Then

(i) every maximal subfield of $D_{n}$ is finitely generated over $k$.

(ii) $k$ is algebraically closed in $D_{n}$; that is, if $\alpha \in D_{n}$ is algebraic over $k$ then $\alpha \in k$.

Proof. (i) We first recall the definition: $A_{n}$ is the $k$-algebra with $2 n$ generators $p_{1}, \ldots, p_{n} ; q_{1}, \ldots, q_{n}$ and relations $\left[p_{i}, q_{j}\right]=p_{i} q_{j}-q_{j} p_{i}=\delta_{i j}$ (Kronecker delta), $\left[p_{i}, p_{j}\right]=0=\left[q_{i}, q_{j}\right]$. We further recall two properties of Weyl algebras: (1) for any $k$-algebra $B, A_{n} \otimes_{k} B \simeq A_{n}(B)$ where $A_{n}(B)$ is the $n$th Weyl algebra with coefficients from $B ;(2) A_{n}(B)$ is Noetherian if and only if $B$ is Noetherian [3, p. 439]. In particular, if $L \subset D_{n}$ is a maximal subfield then $A_{n} \otimes L$ is Noetherian and so also is its partial quotient ring $D_{n} \otimes L$. Finite generation of $L$ follows from Theorem 3(i).

(ii) Let $\alpha \in D_{n}-k$ and put $L=k(\alpha)$. As $D_{n} \otimes L$ is a localization of $A_{n}(L)$, it is a domain. Since $L \otimes L$ embeds in $D_{n} \otimes L$ it also is a domain, and this can hold only if $\alpha$ is transcendental over $k$ [3, p. 248].

It is also known [4] that every maximal subfield of $D_{n}$ has transcendence degree at most $n$ over $k$, but it is not known whether the value is always precisely $n$-this appears to be quite a difficult open question. In addition, it is known that $D_{n}$ contains maximal subfields which are not purely transcendental over $k$. Dixmier has given an example [9, p. 222] of a subfield of $D_{1}$ not purely transcendental over $k$, and a lemma of Roquette [10, p. 209] shows that this field cannot be embedded in any purely transcendental extension of $k$.

Let $L$ be a field of characteristic zero and let $d$ be a nonzero derivation of $L$ with $\operatorname{Ker} d=k$. An Ore domain related to $A_{1}$ is the ring of differential operators over $L, R=L[y ; d][3,12.2]$. In [1], Amitsur proved that the 
centralizer of any $g \in R-L$ is commutative and is a finite free module over $k[g]$. Hence, every maximal commutative subalgebra of $R$ not contained in $L$ is finitely generated over $k$. Let $D$ be the quotient division ring of $R$. It is reasonable to expect an analogous description of the maximal subfields of $D$, although such a result does not seem to be deducible from Amitsur's work on $R$. Instead, we apply Theorem 3.

TheOREM 5. Let $k, L, R, D$ be as in the preceding paragraph. Then the following are equivalent:

(i) $D \otimes D^{0}$ is Noetherian;

(ii) every subfield of $D$ containing $k$ is finitely generated over $k$;

(iii) $L$ is finitely generated over $k$.

Proof. (i) $\Rightarrow$ (ii). Theorem 3.

(ii) $\Rightarrow$ (iii). Clear.

(iii) $\Rightarrow$ (i). Let $A=L \otimes D^{0}$ and extend $d$ to $A$ by putting $d(f \otimes \alpha)=f^{d} \otimes$ $\alpha . A$ is Noetherian by Theorem 3(i) and hence so is $A[y ; d] \simeq L[y ; d] \otimes D^{0}$. As $D \otimes D^{0}$ is a localization of this ring it too is Noetherian.

We remark that a result completely analogous to Theorem 5 holds for quotient rings of twisted polynomial rings of the form $L[x ; \sigma]$ where $L$ is a field extension of $k$ and $c$ is a $k$-automorphism of $L$.

NOTE ADDED IN PROOF. A. Joseph has informed us that he has found an example of a maximal subfield of transcendence degree 1 in the Weyl division ring $D_{2}$, thus settling the question raised after the proof of Theorem 4 .

\section{REFERENCES}

1. S. A. Amitsur, Commutative linear differential operators, Pacific J. Math. 8 (1958), 1-10.

2. N. Bourbaki, Algèbre commutative, Chapitres 1, 2, Hermann, Paris, 1961.

3. P. M. Cohn, Algebra, Vol. 2, John Wiley \& Sons, London-New York, 1977.

4. R. Resco, Transcendental division algebras and simple Noetherian rings, Israel J. Math. (to appear).

5. L. W. Small, A lemma on tensor products of division rings, Pacific J. Math. (to appear).

6. , (unpublished).

7. M. Smith, Centralizers in rings of quotients of group rings, J. Algebra 25 (1973), 158-164.

8. P. Vámos, On the minimal prime ideals of a tensor product of fields, Math. Proc. Cambridge Philos. Soc. 84 (1978), 25-35.

9. J. Dixmier, Sur les algèbres de Weyl, Bull. Soc. Math. France 96 (1968), 209-242.

10. P. Roquette, Isomorphisms of generic splitting fields of simple algebras, J. Reine Angew. Math. 214/215 (1964), 207-226.

Department of Mathematics, University of Southern California, los Angeles, CaliFORNIA 90007 (Current address of Richard Resco)

Department of Mathematics, University of California, San Diego, la Jolla, CaliFORNIA 92093 (Current address of L. W. Small and A. R. Wadsworth) 